\title{
Frontières
}

\section{Sous la férule des fracas}

Penser sous la terreur

\section{Christian Saint-Germain}

Volume 19, numéro 1, automne 2006

Enjeux politiques et mort

URI : https://id.erudit.org/iderudit/016642ar

DOI : https://doi.org/10.7202/016642ar

Aller au sommaire du numéro

Éditeur(s)

Université du Québec à Montréal

ISSN

1180-3479 (imprimé)

1916-0976 (numérique)

Découvrir la revue

Citer cet article

Saint-Germain, C. (2006). Sous la férule des fracas : penser sous la terreur.

Frontières, 19(1), 85-88. https://doi.org/10.7202/016642ar

Ce document est protégé par la loi sur le droit d'auteur. L'utilisation des services d'Érudit (y compris la reproduction) est assujettie à sa politique d'utilisation que vous pouvez consulter en ligne.

https://apropos.erudit.org/fr/usagers/politique-dutilisation/
Cet article est diffusé et préservé par Érudit.

Érudit est un consortium interuniversitaire sans but lucratif composé de l'Université de Montréal, l'Université Laval et l'Université du Québec à Montréal. Il a pour mission la promotion et la valorisation de la recherche. https://www.erudit.org/fr/ 


\section{Sous la férule des fracas Penser sous la terreur}

\section{Christian Saint-Germain, \\ Ph.D., professeur, Département de philosophie, UQAM.}

La situation ne manque pas de pittoresque. Le moment terroriste produit une abondance de colloques pour en déterminer la signification, en absorber le choc, en stabiliser le trauma. Conjointement à l'affolement médiatiquement entretenu de l'opinion publique : le sujet de droit est tout à la fois chauffé à blanc par la menace terroriste et refroidit par l'imminence d'épidémie, voire de pandémie. Cette tenaille permet un déchaînement bureaucratique des polices sanitaires et criminelles. Elle permet, en outre, l'obtention d'un consentement vague mais renouvelé des populations affolées. L'usage de la peur suffit à ce mode de gouvernement invasif. La promesse de sécurité, d'assurance de l'État conduit à une pénétration étatique sans équivalent historique dans toutes les sphères de l'activité individuelle.

La mise en scène médiatique réitérée de la mort violente du citoyen rappelle la fragilité de notions, telles l'État de droit, la liberté d'association ou la délibération démocratique. Un continuum d'intimidation produit l'apparence d'un libre consentement au plan collectif. Thomas Hobbes n'aurait rien pu trouver de mieux pour justifier sa représentation d'un État contraignant et sans contradicteur. La notion de sécurité nationale devenant le concept permettant la subsomption de l'idéal démocratique. Dans le refrain autoritaire, il n'y aurait donc pas de démocratie sans création transnationale de la richesse ni intervention violente contre ceux qui envient l'exercice des libertés individuelles. Le fonctionnement démocratique véritable n'adviendrait que là où le ça théoriste était. Autrement dit, l'individualisme capitaliste ne surgit dans sa forme achevée qu'à la condition de la perpétuation intermittente de l'état de nature. Tout se passe comme s'il fallait susciter ou accepter l'artifice d'un certain désordre pour que le citoyen puisse être amené à prêter allégeance à ceux qui décident déjà des modalités de son apparente liberté de penser, de choisir. L'État, qui devait pourtant s'évanouir à la faveur de la mondialisation - pour faciliter l'application des lois du libre marché - réapparaît pour assurer de la «protection».

Au-delà de cette trop légère introduction sur l'étrangeté du phénomène, nous voudrions tirer toutes les conséquences politiques d'une formule connue de Guy Debord, pour qui :

Cette démocratie si parfaite fabrique elle-même son inconcevable ennemi, le terrorisme. Elle veut, en effet, être jugée sur ses ennemis plutôt que sur ses résultats. L'histoire du terrorisme est écrite par l'État ; elle est donc éducative. Les populations spectatrices ne peuvent certes pas tout savoir du terrorisme, mais elles peuvent toujours en savoir assez pour être persuadées que, par rapport à ce terrorisme, tout le reste devra leur sembler plutôt acceptable, en tout cas plus rationnel et plus démocratique (Debord, 1979, p. 40).

Si l'histoire du terrorisme est éducative, écrite par l'État, il faut donc comprendre que ce terrorisme «n'est pas ce qu'il prétend être». Qu'il est essentiellement de nature pédagogique. L'État moderne instruit les masses sous la férule des fracas et les place devant leur unique requête: le désir d'être protégées. Cette stratégie des pouvoirs publics commande une obédience des sujets d'un genre nouveau. Une solidarité individuelle, désinformée jusque dans la mort, le fait de vivre en panique, en état de choc si l'on entend par panique «une absence totale de coordination, de coopération, de concertation, entre les actions qui deviennent soumises à une logique purement individualiste» (Dupuy, 1991, p. 32).

Pour conserver la forme exquise de cette docilité, l'État revêt ses habits archaïques, rappelle la nécessité du monopole de la violence, du sien qui ne souffre de concur- rent qu'aux dépens des majorités silencieuses. L'État moderne apparaît donc à l'occasion de la terreur sous des traits anciens, pré-juridiques : féodaux et mafieux. Il suppose la démonstration constante que l'ordre est établi sur la violence et que sans elle, sans la contrainte, l'étroite surveillance, l'assujettie est rappelée à la régression dans la plus complète impuissance. Cette technique oscille entre la sidération ordinaire du citoyen planté devant son téléviseur et l'écarquillement brutal de cette somnolence.

Les politiques de santé et celles contre le terrorisme sont les deux faces d'une relation d'emprise bifide. Assurer par tous moyens la sécurité de chacun, faire le bonheur du plus grand nombre. Cette perspective résume le projet politique de la modernité. Elle implique pour se réaliser tout un «savoir inquiéter » et une "sollicitude comptable» au soutien de ces simulacres humanistes. Une bio-politique qui génère, en feignant de les subir, ses ennemis - viraux ou terroristes -, les appuie contre de complexes échafaudages bureaucratiques. Nous voudrions décrire ce monstre bicéphale: l'intrication d'un État de terreur et d'un État de santé. La double consistance d'un même totalitarisme. Aucune contradiction dans les finalités de ces relations d'emprise. Simplement leur cohabitation qui justifie l'excroissance, la prolifération de moyens orthopédiques de toute nature : l'institutionnalisation de procédures invasives exercée sur un sujet devenu - tant du point de vue politique que biologique - génétiquement translucide, prévisible.

Par l'établissement de défense invasive, l'envahissement du for intérieur des sujets, c'est tout un système de direction assistée des masses qui affirme sa préséance. Par l'imposition de ce régime de protection et le spectacle permanent des menaces diverses, le citoyen est amené à une complète soumission à un système qui le calcule vivant et mort ou tout à la fois mort-vivant: déjà mort à sa naissance, compté pour mourant 
dans l'extase statistique qui signe la complète insignifiance de la vie humaine.

Plus encore, la mise en place de ce dispositif de prise en charge sonne la fin de la contestation qui se trouve anéantie comme par prétérition. Comment résister, s'opposer à un système qui ne prétend pourvoir qu'à la sécurité de tous, à l'éradication des maladies mortelles? Comment concevoir un démenti collectif devant ce qui ne cesse de susciter la participation à ce bien-être ? L'appartenance à ce dispositif implique plutôt l'obligeance infinie dans la dépendance de chacun aux mailles d'un réseau intelligent. Il provoque rien de moins que la suppression irrévocable de toute idée de substitution de cette politique même. Il est difficile d'imaginer que l'on puisse encore, à notre époque, penser à l'extérieur de cette boîte - out of the box -, que sous le mode de l'intensification ou de la modération de cet état de fait ${ }^{1}$.

C'est que vivre en réseau implique nécessairement de vivre sous la menace d'un retour de flamme. Cette appartenance ne confère au sujet aucune autre réalité que celle de se trouver inclus dans un trajet. Elle implique en outre de laisser venir vers soi une multitude de tribulations et d'admettre la réversibilité de ce qui y circule. Cet acquiescement involontaire constitue notre immersion totale dans le destin des technologies et l'impossibilité d'en sortir autrement qu'au sein des réalisations de celles-ci. Comme par l'effet de la réversibilité, de l'inversion maligne de la commodité, le moindre événement, la moindre erreur dans la manipulation des stocks vivants portent des conséquences incalculables.

Pourtant par le plus curieux agencement des pôles sociaux, la modernité ne cesse «d'assurer » le citoyen de sa longévité sous l'égide d'un État tout tourné vers des calculs de santé. La prévoyance actuarielle emmaillote d'une inquiétante tranquillité le malheureux bénéficiaire. Une immense rhétorique centrée sur la notion de «qualité de vie» et le calfeutrage financier des destins individuels cohabitent avec la mort subite sous la terreur. Un peu comme dans la méthode policière d'interrogatoire à deux agents: good cop, bad cop, la mise en scène de ces alternatives à l'occasion du terrorisme ou de la menace virale pandémique fait des merveilles pour confondre les récalcitrants. Dans une existence dont l'échéance actuarielle est inscrite, la qualité de vie mesurée, les éléments de disruption exercent inconsciemment aussi une attraction jubilatoire.

Alexis de Tocqueville notait dans la quatrième partie De la démocratie en Amérique:

Je veux imaginer sous quels traits nouveaux le despotisme pourrait se produire dans le monde: je vois une foule innombrable d'hommes semblables et égaux qui tournent sans repos sur

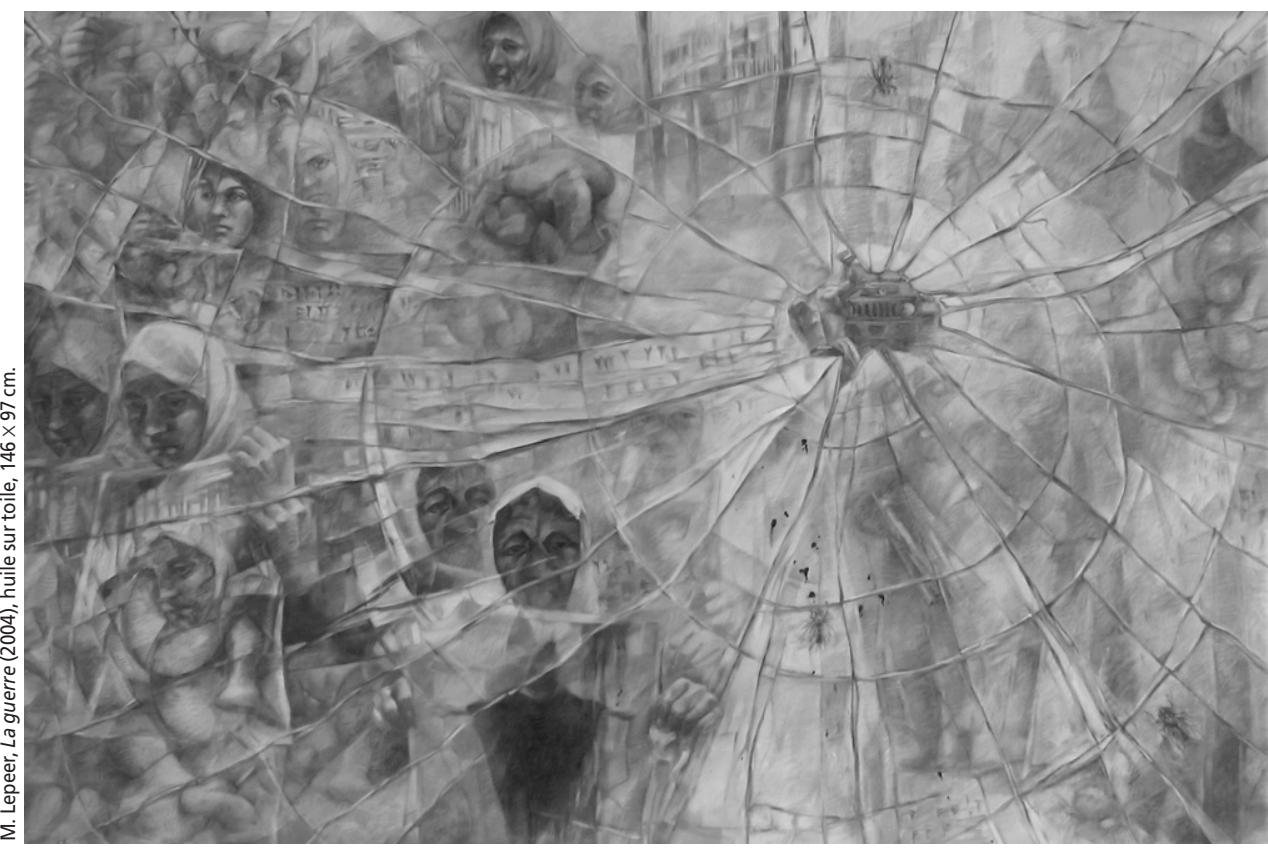

eux-mêmes pour se procurer de petits et vulgaires plaisirs dont ils emplissent leur âme. Chacun d'eux retiré à l'écart, est comme étranger à la destinée de tous les autres [...] Au-dessus de ceux-là s'élève un pouvoir immense et tutélaire, qui se charge seul d'assurer leur jouissance et de veiller sur leur sort. Il est absolu, détaillé, régulier, prévoyant, doux. [...] Il aime que les citoyens se réjouissent, pourvu qu'ils ne songent qu'à se réjouir. Il travaille volontiers à leur bonheur ; mais il veut en être l'unique agent et le seul arbitre; il pourvoit à leur sécurité, prévoit et assure leurs besoins, facilite leurs plaisirs [...] que ne peut-il leur ôter entièrement le trouble de penser et la peine de vivre? (Pontalis, 1982, p. 5.)

Toute se passe donc depuis cette alternative de prise en charge et de mise à l'abri, de la solidarité mortelle de tous et du désir de sécurité. Cette alternance reconfigure l'économie psychique des sujets en les faisant tantôt passer de l'exubérance consumériste à l'imminence du simple état de subsistance.

La construction médiatique d'une fébrilité artificielle et la désillusion fonctionnent au sein d'une architecture de la menace, du danger. Stratégie qui maintient dans un bain d'images des sujets isolés - en particules d'opinions - mais aussi sans possibilité de distanciation, tenus en joue par l'appel d'air des effondrements. Il suffit ensuite de remplacer les lambeaux du sentiment d'appartenance à une société technologique par un syndrome de Stockholm généralisé. Ce tour de force suppose de prendre les populations spectatrices en otage, de les maintenir en permanence dans ce statut. D'obtenir par là, l'adhésion au diktat d'une aristocratie monétaire et technologique.
Cette transaction à laquelle le sujet déboussolé prête allégeance n'est pas l'un des moindres moments pour imposer l'horizon unique d'un état totalitaire. Une spirale législative s'engage donc autour du thème "assurer la sécurité". Sollicitude à l'égard des populations qui n'a d'égale que la puissante diversion qu'elle opère sur les véritables menaces planétaires, les réchauffements et désertifications multiples. Dans une telle situation par exemple, la police londonienne prévient qu'elle «tire pour tuer» ceux qui ne s'arrêtent pas. Comme si les choses étaient différentes du temps ordinaire. Mais un policier qui n'exerce pas sa discrétion, qui «tire pour tuer», sans s'être trouvé menacé, est-il si différent d'un gardien dans un camp de concentration?

Pour les populations, écrit Debord, «par rapport à ce terrorisme, tout le reste devra leur sembler plus acceptable». Cette assertion contient toute la mécanique d'intimidation hobbesienne, l'imminence de la ruine, de la déperdition probable s'il advenait que cet État totalitaire défaille. Dès le lendemain des événements londoniens, les travailleurs salariés se précipitent à nouveau dans les tunnels, s'entassent dans les métros marquant une solidarité dans la mort qui présage la totale plasticité des masses. Un peu comme à l'occasion des premiers accidents de travail de la société industrielle qui donnaient lieu à de chaudes réconciliations des travailleurs et patrons au-dessus des cercueils ouverts. Par le plus curieux revirement, les sociétés réclament l'amputation des principes démocratiques, l'atténuation des libertés fondamentales, croyant conjurer ainsi, par automutilation collective, des menaces erronément attribuées au monde extérieur. Baudrillard décrit cette régression en ces termes: 
Au point que l'idée de liberté, idée neuve et récente, est déjà en train de s'effacer des mœurs et des consciences, et que la mondialisation libérale est en passe de se réaliser sous la forme exactement inverse : celle d'une mondialisation policière, d'un contrôle total, d'une terreur sécuritaire. La dérégulation finit dans un maximum de contraintes et de restrictions, équivalant à celle d'une société fondamentaliste (Baudrillard, 2002, p. 43).

Il ne s'agit pas de lire les attentats $\mathrm{du} 11$ septembre dans une perspective hollywoodienne, mais d'y suivre le destin de l'être exposé, du sujet moderne. D'y surprendre une arme de «persuasion massive» pour les récalcitrants de la société technologique. La mise en place d'un artifice violent et, partant, la mise au pas citoyenne à une allégeance féodale au destin des groupes: le déchaînement d'un État policier. Baudrillard ajoute que:

Du moment où les États ne peuvent plus s'attaquer ou se détruire les uns les autres, ils se retournent presque automatiquement vers leur propre peuple ou leur propre territoire, dans une sorte de guerre civile, intestine, de l'État contre sa propre référence naturelle (Baudrillard, 1990, p. 85).

Une folie policière auto-immune. L'exposition d'innocents à la force de rachat d'un système qui se dédouane de sa violence à venir. Il n'est donc pas question de soutenir que les événements du 11 septembre n'ont pas eu lieu ou, encore moins, qu'ils obéissent à la part maudite d'un système si parfait qu'il sécrète son double désordonné par surabondance d'ordre. Plutôt, de supposer un engrenage policier, de suggérer une théorie restreinte du complot impliquant des noyaux fonctionnarisés dans l'État moderne qui le dirige comme autant de balises internes vers d'autres horizons. Manière de dire que sous la représentation démocratique - et donc sans égard pour la personnalité des dirigeants -, une autre mécanique laisse, tantôt agir, tantôt retient des membres d'organisations authentiquement hostiles à l'Amérique ou à l'Europe, mais dont la plupart des actions d'éclat sont prévisibles. Il n'y a pas de corps étrangers du point de vue des réseaux de police, de leur force d'immunité moderne. D'ailleurs dès le lendemain d'un acte terroriste, les quotidiens montrent en première page la tronche pileuse des suspects des mandats internationaux d'arrestation ayant vite été émis. Accepter la version officielle fournie par les médias reviendrait à méconnaître sciemment la puissance d'infiltration, d'information des polices modernes et faire fi du fait que:
Tout individu notoirement ennemi de l'organisation sociale ou politique de son pays, et, davantage encore, tout groupe d'individus contraint de se déclarer dans cette position adverse est connu de plusieurs services de renseignements concurrents

(Bounan, 2003, p. 18).

S'installe donc une zone policière indiscernable qui, au prétexte de lutter efficacement contre la menace, se voit octroyer des pouvoirs qui la placent de plain-pied dans l'immunité juridique. Une "police criminelle», qui se comporte comme telle sans jamais devoir répondre de ses actes. Une structure opérationnelle qui ne s'embarrasse plus de mandat, puisque le scénario suggère un état de guerre interne, internalisée. pouvoirs publics qui peuvent colliger sur lui archives et observations cachées, l'épier à l'occasion de conversations téléphoniques ou d'emprunts de livres dans les bibliothèques publiques. Depuis le 11 septembre, de nombreux commentateurs ont conclu que l'acuité stratégique de cette attaque forçait à considérer que désormais: "Tout est possible. » Or, n'était-ce pas une expression, un sentiment éprouvé par les survivants des camps de concentration? Une préparation à la mise en place de mesures insolites, disproportionnées obligeant chacun à un credo quia absurdum politique?

Le citoyen est ainsi tenu en otage entre les menaces apparemment malicieuses de groupes subversifs et placés devant le danger de pandémie, d'extermination subite

\section{AFIN QU'IL PUISSE ACCEPTER L'ORDRE ÉCONOMIQUE,}

\section{FAUT-IL QUE LE CITOYEN SOIT PONCTUELLEMENT RECONDUIT}

À UN ÉTAT DE PRÉCARITÉ?

Tout se passe comme si le sujet se faisait rappeler, au cœur du divertissement spectaculaire, l'existence d'une "économie psychique de subsistance», son propre Ground Zero. L'abondance des itinérants dans les grandes villes sert efficacement cette technique pédagogique. Qu'il peut, à tout moment, être réduit à sa plus simple expression démocratique, à un état de précarité économique ou même sur le point de devenir un ennemi politique potentiel. Or, il n'existe plus de politique si l'on entend par là la libre discussion d'idées complexes, impliquant l'exercice d'une liberté pardessus le marché planétaire. Le scénario terroriste doit bien porter sur autre chose, attenter à la capacité de penser elle-même. À moins justement que la finalité de l'entreprise véritable implique la mise à mort psychique du sujet? L'écrivain Bernard Noël observe que:

Le pouvoir a compris depuis longtemps que censurer la liberté d'expression n'était qu'un pis-aller et que, pour être efficace, il lui fallait s'attaquer à la liberté de penser, c'est-à-dire au lieu qui, en chacun de nous, est la matrice du sens (Noël, 1994, p. 107).

Afin qu'il puisse accepter l'ordre économique, faut-il que le citoyen soit ponctuellement reconduit à un état de précarité? À tâter la médecine de cheval des lois d'exception comme le Patriot Act ou ses succédanés canadiens qui "détruisent la démocratie au prétexte de la défendre» selon la belle expression du juriste Pierre Robert. Un sujet à découvert, devenu complètement transparent, nu au regard des par virus ou même par bio-terrorisme. Une rhétorique de la contagion et de la contamination tient chaque atome individuel en sursis, dans la suspicion réciproque. C'est qu'à l'instar des terroristes, les virus ne sont guère inquiétés par la légendaire sagacité des douaniers. Désintégré nationalement, l'espace social est alors compris dans l'admission des effondrements immunitaires. Des populations entières sont alors exposées comme elles l'étaient lors de la guerre froide, non plus seulement à la faveur de crises politiques ponctuelles, mais continûment, à la peur. Soumises à la peur, par la peur? Jean-Paul Curnier décrit en ces termes, le paysage mental à l'intérieur duquel la modernité a lieu :

L'homme moderne marche à la peur.

Je ne sous-entends pas qu'il n'y ait aucune raison d'avoir peur, de la guerre, du chômage, du Sida ou du délabrement de l'environnement, j'affirme simplement ceci : que ces menaces, parce qu'elles s'exercent sur un monde affolé et comme prédisposé à la terreur, loin d'être affrontées comme telles, participent d'un affaiblissement plus fort encore (Curnier, 2002, p. 25).

Mais «avoir peur» n'est peut-être que le dernier mécanisme qui assure chacun de la réalité de son existence. Naître aujourd'hui implique pour le vivant humain de grandir à l'ombre de sa disparition pressentie, les yeux rivés aux indices de $\mathrm{CNN}$ colorant le degré de sévérité de menaces pesant sur les grandes villes américaines. De penser sous la terreur à la réversibilité maligne des dépendances à autrui et à l'ordre technologique. 
La ville n'y a plus la figure du rempart dressé contre l'invasion, elle est le piège qui se referme sur la masse, elle devient la barbarie avec un autre visage. La ville une agglomération offerte à la destruction massive. L'agglomération ne peut produire que la majorité silencieuse

(Sheer, 1978, p. 63).

La question n'est plus alors la détermination des conditions politiques du vivre en commun mais comment échapper à la contention des villes. De découvrir en panique que ce que l'on croyait être une communauté devient de fait une fosse commune, un site d'enfouissement sanitaire. Ne resterait-il d'intérêt politique dans la modernité qu'à imaginer des plans d'évacuation, des sorties de secours?

Tels des jumeaux identiques, virus et terroristes marquent l'infiltration de l'insensé dans le dispositif de planification rationnelle des sociétés de consommation avancées. Insensé parce qu'il ouvre à une dimension de l'irrécupérable, de l'inassimilable à la logique d'une reconnaissance ou d'une domination finale éventuelle. Bulle de pure perte dans le cocon des tranquillités consuméristes, il n'est guère possible de conjurer l'apparente survenue de l'aléa, de la force majeure ou du cas fortuit. À l'encontre de la peur construite, de l'état de panique entretenu, une étrange disposition psychologique de chacun: une insouciance généralisée - autre versant sans doute -, de la panique, suggérant au citoyen de s'imaginer introuvable, perpétuellement à l'abri devant son téléviseur. Attitude confortée par le fait qu'un grand nombre de catastrophes sont retransmises en direct. Cette situation renvoie chacun à un comportement connu des survivants des camps qui ne pouvaient s'empêcher d'éprouver un sentiment de délivrance coupable lorsqu'il assistait, sans en être victime, à la molestation d'un codétenu.

Pourtant, ailleurs n'a jamais été aussi près, et bientôt, presque sans référence à quelque éloignement géographique. Cette ambivalence morale du survivant tranche avec toutes autres formes de désillusions métaphysiques. Elle est la condition morale - hautement ambiguë - du sujet moderne. Ni coopération directe, ni sympathie - simplement la conviction silencieuse que, notre numéro d'assurance sociale dans cette loterie insolite, notre matricule peut à tout moment être pigé.

Ces états de détresse ont fait l'objet d'observations psychologiques. Des couples de voyageurs survivants, soumis à l'imminence d'un crash en avion se séparent - après les événements - après avoir découvert la vraie nature de leur conjoint (Bauman, p. 29). Surpris par l'autre qu'il croyait connaître, par son insouciance égoïste, la froideur manifestée. Les individus ne peuvent malheureusement pas divorcer de leur masse de référence.

Même le bienveillant Jean-Jacques Rousseau n'a pas manqué, à son époque, de remarquer cette propriété des groupes humains à s'exciter du malheur d'autrui :

Qu'on admire tant qu'on voudra la société humaine, il n'en sera pas moins vrai qu'elle porte nécessairement les hommes à s'entre-haïr à proportion que leurs intérêts se croisent, à se rendre mutuellement des services apparents et à se faire en effet tous les maux imaginables. [...] Il n'y a peut-être pas un homme aisé à qui des héritiers avides et souvent ses propres enfants ne souhaitent la mort en secret, pas un vaisseau en mer dont le naufrage ne fût pas une bonne nouvelle pour quelque négociant, pas une maison qu'un débiteur ne voulût voir brûler avec tous le papiers qu'elle contient; pas un peuple qui ne se réjouisse des désastres de ses voisins. [...] J'ai vu des hommes affreux pleurer de douleur aux apparences d'une année fertile, et le grand et funeste incendie de Londres, qui coûta la vie ou les biens à tant de malheureux, fit peut-être la fortune à plus de dix milles personnes (Rousseau, 1971, p. 251-252). Le sujet de droit entre dans l'ère des extinctions subites. Jamais n'aura-t-il autant été mis en garde, rappelé à l'insécurité foncière d'appartenir à un groupe donné. Il aura aussi été habitué à assister impuissant à la mort d'autres groupes particuliers dans des populations lointaines. Cette habitude de voir mourir en masse, d'admettre la catastrophe comme composante inséparable de notre mode de vie est ce qui donne le plus à penser. Or, il n'est plus sûr que même un citoyen averti puisse accepter la somme des conséquences immorales présupposées par son existence immédiate. L'accident viral ou l'incident terroriste marque notre appartenance à cette "pédagogie de la catastrophe» en forçant à ne vivre les effets de la solidarité que sous le mode du pâtir. Comme si toujours, une terreur diffuse ne demandait qu'à se fixer à un contenu, à s'approprier une menace.

Un invivable tiraillement surgit, tout accaparé par l'impératif néolibéral du "chacun pour soi», et, dans un même moment, par son impossibilité même par dépendance extrême, structurelle, à autrui. $\mathrm{Ni}$ avec ni sans. Une double contrainte qui se défait violemment dans la dissolution du rêve individuel et le réveil brutal au cauchemar collectif. Violence de la panique tout autant que de l'objet de la menace. Dans Masse et Puissance, Élias
Canetti décrit la force subite qu'exerce cette désillusion:

Dans un théâtre, on l'a souvent remarqué déjà, la panique est une désintégration de la masse. Plus les gens étaient soudés par la représentation, plus était fermée la forme du théâtre qui les contenait extérieurement, et plus la désintégration est violente (Canetti, 1960, p. 24).

Depuis l'entrée dans l'âge atomique la possibilité de recevoir la mort d'une malversation policière ou d'un lapsus technologique rassemble, en le surexposant, le genre humain. Nous sommes collectivement en situation de perpétuelle ordalie. Et à l'instar des vieux textes bibliques qui empoisonnent toujours l'imaginaire collectif, une destruction totale paraît le seul véritable horizon historique. Peut-être faudrait-il imaginer une seule mesure pour notre condition, celle du degré d'impuissance de chacun sous la tutelle des technologies. Décrire ensuite la physique de l'atome individuel dans l'accélérateur de catastrophes qu'est devenue toute grande ville.

\section{Bibliographie}

BAUDRILLARD, J. (2002). L'esprit du terrorisme, Paris, Galilée.

BAUDRILLARD, J. (1990). La transparence du mal. Essai sur les phénomènes extrêmes, Paris, Galilée.

BAUMAN, Z. (1989). Modernité et holocauste, Paris, Éditions La Fabrique.

BOUNAN, M. (2003). Logique du terrorisme, Paris, Allia.

CANETTI, E. (1960). Masse et puissance, Paris, Gallimard.

CURNIER, J.-P. (2002). Aggravation 19892001, Paris, Éditions Léo Sheer.

DEBORD, G. (1979). Commentaires sur la société du spectacle, Paris, Gallimard.

DUPUY, J.-P. (1991). La panique, Paris, Les empêcheurs de penser en rond.

NOËL, B. (1994). La castration mentale, Paris, Ulysse fin de siècle.

PONTALIS, J.-B. (dir.) (1982). Le trouble de penser, Nouvelle revue de psychanalyse, Paris, Gallimard, $\mathrm{n}^{\circ} 25$.

ROUSSEAU, J.-J. (1971). Euvres complètes 2, Euvres philosophiques et politiques: des premiers écrits au Contrat social 1735-1762, Paris, Seuil.

SHEER, L. (1978). La société sans maître, Paris, Galilée.

\section{Note}

1. À moins que l'apparente obsession pour la sécurité sanitaire ou la lutte antiterroriste ne cache le troisième terme de cette équation : la situation véritable des écosystèmes. 